\title{
Optical Magnification Should Be Mandatory for Microsurgery: Scientific Basis and Clinical Data Contributing to Quality Assurance
}

\author{
Harald Schoeffl ${ }^{1,2}$, Davide Lazzeri ${ }^{2,3}$, Richard Schnelzer ${ }^{1,2}$, Stefan M. Froschauer ${ }^{1,2}$, \\ Georg M. Huemer ${ }^{2,3}$ \\ ${ }^{1}$ Department of Trauma Surgery, General Hospital Linz, Linz; ${ }^{2}$ maz - Microsurgical Training Center, Linz; ${ }^{3}$ Section of Plastic and \\ Reconstructive Surgery, General Hospital Linz, Linz, Austria
}

Background Microsurgical techniques are considered standard procedures in reconstructive surgery. Although microsurgery by itself is defined as surgery aided by optical magnification, there are no guidelines for determining in which clinical situations a microscope or loupe should be used. Therefore, we conducted standardized experiments to objectively assess the impact of optical magnification in microsurgery.

Methods Sixteen participants of microsurgical training courses had to complete 2 sets of experiments. Each set had to be performed with an unaided eye, surgical loupes, and a regular operating microscope. The first set of experiments included coaptation of a chicken femoral nerve, and the second set consisted of anastomosing porcine coronary arteries. Evaluation of the sutured nerves and vessels were performed by 2 experienced microsurgeons using an operating microscope.

Results The 16 participants of the study completed all of the experiments. The nerve coaptation and vascular anastomoses exercises showed a direct relationship of error frequency and lower optical magnification, meaning that the highest number of microsurgical errors occurred with the unaided eye. For nerve coaptation, there was a strong relationship $(\mathrm{P}<0.05)$ between the number of mistakes and magnification, and this relationship was very strong $(P<0.01)$ for vascular anastomoses.

Conclusions We were able to prove that microsurgical success is directly related to optical magnification. The human eye's ability to discriminate potentially important anatomical structures is limited, which might be detrimental for clinical results. Although not legally mandatory, surgeries such as reparative surgery after hand trauma should be conducted with magnifying devices for achieving optimal patient outcomes.

Keywords Microsurgery / Training / Optical magnification / Microscope / Surgical loupes
Correspondence: Georg M. Huemer Section of Plastic and Reconstructive Surgery, General Hospital Linz, Krankenhausstrasse 9, 4020 Linz, Austria

Tel: $+43-732-780673819$

Fax: $+43-732-78063190$

E-mail: ghuemer@drhuemer.com
No potential conflict of interest relevant to this article was reported.

\section{INTRODUCTION}

The advent of microsurgery in the 1960's is considered to be one of the most important milestones in recent plastic and reconstructive surgery [1]. Although there is no generally agreed definition, microsurgery may be defined as surgery requiring an

Copyright $(92013$ The Korean Society of Plastic and Reconstructive Surgeons

This is an Open Access article distributed under the terms of the Creative Commons Attribution Non-Commercial License (http://creativecommons.org/

licenses/by-nc/3.0/) which permits unrestricted non-commercial use, distribution, and reproduction in any medium, provided the original work is properly cited. 
operating microscope. The first to coin the term "microsurgery" was the vascular surgeon, Jules Jacobson, who anastomosed blood vessels with a diameter smaller than $1.4 \mathrm{~mm}$ with the aid of a microscope [2]. Nowadays many surgical subspecialties, such as plastic surgery, trauma surgery, neurosurgery, and maxillofacial surgery, use microsurgery in their clinical routine. Successful microsurgical operations require sufficient training and experience using delicate instruments and suture material of $8 / 0$ and less. In addition, optical magnification should be mandatory for precise handling of tissue and sutures. Optical magnification can be achieved using loupes [3] or regular operating microscopes [4]. However, optical magnification may be neglected for several reasons such as lack of availability of devices, laborious preparation of the operating microscope, or a surgeon's unwillingness to use an operating microscope. During a surgical procedure, in which certain delicate structures such as peripheral nerves are involved, it is not legally mandatory to use optical magnification in the form of loupes or an operating microscope. Nevertheless, exact approximation of neural or vascular tissues is imperative for a successful microsurgery.

The purpose of this paper is to objectively evaluate the impact of optical magnification on the success of microsurgery. The presented results should serve as a guideline for the novice microsurgeon on when to use optical magnification in various clinical situations.

\section{METHODS}

\section{Neurophysiological fundamentals}

The eye has a visual acuity threshold below which an object will go undetected. The standard definition of normal visual acuity (20/20 vision) is the ability to resolve a spatial pattern separated by a visual angle of one minute of arc (MOA). Because one degree contains 60 minutes, a visual angle of one MOA is $1 / 60$ of a degree. Furthermore, one MOA equals 60 arcseconds. The spatial resolution limit is derived from the fact that one degree of a scene is projected across $288 \mu \mathrm{m}$ of the retina by the eye's lens. In this $288 \mu \mathrm{m}$, there are 120 color sensing cone cells packed. Thus, if more than 120 alternating white and black lines are crowded side-by-side in a single degree of viewing space, they will appear as a single gray mass to the human eye.

Microsurgical suture material of size $11 / 0$ presents a thread thickness of $50 \times 10^{-6} \mathrm{~m}$. For instance with a working distance of $50 \mathrm{~cm}$, the thread is depicted on the retina with a visual angle of 20.6 arcseconds according to the following formula: alpha [degree] $=(180 / \mathrm{Pi}) \times \arctan \left(50 \times 10^{-6} / 0.5\right)$. Considering solely the optical abilities of the human eye, the $11 / 0$ thread may be impossible to visualize. The thread is merely visible because it is contrasted, creating a diffraction/flexion phenomena, which are perceptible as a misalignment (Vernier acuity). Vernier acuity refers to the ability to determine when two parallel straight lines are exactly in line [5]. The perceptibility depends on the brightness, wavelength, place, size, and duration of the manipulation as well as of the retinal adaptation. The contrast sensitivity of the human eye depends on the light-dark changes per visual angle.

A $\times 6$ magnification would lead to an image or depiction of the above-mentioned thread on the retina with a visual angle of 120 arcseconds, which is sufficient for a purely optical perception. Thus, the discrimination of two structures lying next to each other is made possible, and consequently, the surgical manipulations are under absolute visual control.

\section{Practical experiments}

The authors are members of maz, which is a microsurgical training and research center. Persons with an interest in microsurgery are trained at various levels of expertise, starting from complete novices, to participants who have had some exposure to microsurgery learning basic microsurgical techniques, to medical doctors in a surgical specialty dealing with various microsurgical problems learning operative dissection. In this study, during the basic microsurgical courses, 16 voluntary participants (age range, 22 to 28 years old; mean, 25.8 years) with comparable levels of expertise in surgery were asked to perform the following experiments.

\section{Experiment 1}

In the first experiment, the participants had to coapt an intentionally cut ischiadic nerve $3 \mathrm{~cm}$ distal to the femoral head on the chicken leg $[6,7]$ without using a magnifying optical device (exercise 1), with $2.5 \times$ magnifying glasses (EyeMag Smart, Zeiss, Oberkochen, Germany) (exercise 2), and with an operating microscope (OPMI pico, Zeiss) (exercise 3). The mean diameter of the chicken nerve at the thigh level was $1.28 \pm 0.13$ $\mathrm{mm}$. The magnification of the microscope could be adjusted randomly but had to be above $6 \times$ magnification. The exercise, which had to be performed first, was randomized by lot. Standard microsurgical instruments (Aesculap, Tuttlingen, Germany) from our training courses and suture material 10/0 (Monosof, Covidien, Dublin, Ireland) were used. All coaptations had to be carried out using interrupted stitches. The specimen (chicken legs), with a weight of 248 to $291 \mathrm{~g}$ (average, $273 \mathrm{~g}$ ), were purchased from a supermarket. Chicken legs are a standard training model in our center [8].

\section{Experiment 2}

In the second experiment, the participants had to anastomose 
a porcine coronary vessel 7-9 with a vascular lumen of approximately $1.5 \mathrm{~mm}$. For all of the anastomosis, interrupted stitches had to be used. Again, the end-to-end anastomosis was performed without a magnifying optical device (exercise 1), with 2.5 magnifying glasses (exercise 2 ), and with an operating microscope (exercise 3 ). The magnification of the microscope could be adjusted randomly but had to be above $6 \times$ magnification Which exercise among the three had to be performed first was randomized by lot. Standard microsurgical instruments from our training courses and suture material 9/0 (Monosof, Covidien) were used. The pig hearts were purchased fresh and unopened from a slaughterhouse and had a weight of 353 to $487 \mathrm{~g}$ (average, $405 \mathrm{~g}$ ). Pulsatile perfused pig heart coronary vessels are a standard training model in our center [9].

\section{Assessment}

Evaluation of the sutured nerves and vessels was performed by 2 experienced microsurgeons using an operating microscope. Each mistake was counted only once for each participant. The specimens (chicken legs and porcine hearts) were blinded to the evaluators by two persons not involved in the experiment or evaluation. The anastomoses were cut open to be examined on all of the sides, and the nerve was also dissected. Mistakes were noted and documented with a digital camera connected to the microscope.

The types of mistakes collected after the nerve repair included (experiment 1): 1) Insertion depth: an insertion depth mistake means that not only the epineurium but also the axon was captured with the stitch; 2) Rotation: there is a noticeable rotational mistake in aligning the ends of the cut nerve; 3) Nerve damage through pressure and suture asymmetry: there is noticeable damage in the integrity of the nerve through inadequate forceps pressure or misalignment of the interrupted sutures.

On the other hand, the types of mistakes recorded after porcine coronary vessel anastomosis exploration included (experiment 2): 1) Backwall sutures: any stitches that captured the opposing backwall and thereby occluded the lumen of the vessel;
2) Distance between stitches and leakage: distances between each interrupted stitch that are too wide and will cause leakage; leakage was checked separately by perfusing the vessel with an ink-colored fluid; 3) Suture asymmetry and rotation: visible difference between the edges of the vessel that were captured within a single interrupted suture, and a gross rotational mistake causing kinking of the vessel; 4) Vessel damage through pressure: noticeable alterations of the integrity of the vessel wall through pressure injury caused by forceps.

\section{Statistical analysis}

Statistical analysis was performed using the McNemar test to compare the unadjusted categorical data of the exercise groups obtained during the two experiments. All of the tests were twosided. The differences were considered statistically significant at $\mathrm{P} \leq 0.05$.

\section{RESULTS}

All of the 16 participants completed all the necessary experiments. The results for each experiment for the 16 students are given in Tables 1, 2.

By choosing 16 voluntary participants of the basic microsurgical courses with comparable surgical levels, we were able to minimize the surgical-related variables in performing the described experiments.

\section{Experiment 1 group}

Within this group, the number of mistakes made during the three exercises (without a magnifying optical device, with $\times 2.5$ magnifying glasses, and with an operating microscope) did not differ significantly in rotation, nerve damage through pressure, or suture asymmetry. On the other hand, comparisons across the outcomes obtained within the three types of exercises yielded significant differences in insertion depth, with far greater superiority of the operating microscope over the surgical loupes and simple naked-eye sutures $(\mathrm{P}<0.01)$. Finally, the analysis showed

Table 1. Overview and comparison of results and for 3 sets of experiments for nerve coaptation

\begin{tabular}{|c|c|c|c|c|c|c|c|c|c|}
\hline \multirow{2}{*}{ Type of mistake } & \multicolumn{3}{|c|}{ WM \& SL } & \multicolumn{3}{|c|}{ WM \& OM } & \multicolumn{3}{|c|}{ SL \& OM } \\
\hline & WM & SL & P-value ${ }^{\text {a) }}$ & WM & OM & P-value ${ }^{\text {a) }}$ & $\mathrm{SL}$ & OM & P-value ${ }^{\text {a) }}$ \\
\hline Insertion depth & 16 & 11 & 0.0625 & 16 & 3 & 0.0002 & 11 & 3 & 0.0078 \\
\hline Rotation & 3 & 1 & 0.50 & 3 & 1 & 0.50 & 1 & 1 & - \\
\hline Nerve damage through pressure & 5 & 2 & 0.25 & 5 & 2 & 0.25 & 2 & 2 & - \\
\hline Suture asymmetry & 11 & 9 & 0.50 & 11 & 9 & 0.50 & 9 & 9 & - \\
\hline No. of accurate specimen & 0 & 3 & 0.20 & 0 & 10 & 0.002 & 3 & 10 & 0.0156 \\
\hline
\end{tabular}


Table 2. Overview and comparison of results for 3 sets of experiments for vessel anastomosis

\begin{tabular}{|c|c|c|c|c|c|c|c|c|c|}
\hline \multirow{2}{*}{ Type of mistake } & \multicolumn{3}{|c|}{ WM \& SL } & \multicolumn{3}{|c|}{ WM \& OM } & \multicolumn{3}{|c|}{ SL \& OM } \\
\hline & WM & SL & P-value ${ }^{a)}$ & WM & OM & P-value ${ }^{a)}$ & SL & $\mathrm{OM}$ & P-value ${ }^{a)}$ \\
\hline Backwall suture & 9 & 6 & 0.25 & 9 & 1 & 0.0078 & 6 & 1 & 0.0625 \\
\hline Distance between stitches & 14 & 8 & 0.0313 & 14 & 7 & 0.0156 & 8 & 7 & 1 \\
\hline Leakage & 8 & 6 & 0.50 & 8 & 5 & 0.25 & 6 & 5 & 1 \\
\hline Rotation & 2 & 0 & 0.50 & 2 & 0 & 0.50 & 0 & 0 & - \\
\hline Vessel damage through pressure & 6 & 2 & 0.125 & 6 & 2 & 0.125 & 2 & 2 & - \\
\hline Suture asymmetry & 9 & 3 & 0.0313 & 9 & 3 & 0.0313 & 3 & 3 & - \\
\hline No. of accurate specimen & 0 & 4 & 0.25 & 0 & 12 & 0.001 & 4 & 12 & 0.0156 \\
\hline
\end{tabular}

a significant difference in the number of accurate neurorrhaphies of the nerve stumps in favor of the operating microscope over the surgical loupes $(\mathrm{P}<0.01)$ and sutures without magnification $(\mathrm{P}<0.01)$ (Table 1).

\section{Experiment 2 group}

Leakage, rotation, and vessel damage through pressure did not differ significantly among the three sets of experiments for vessel sutures. Anastomosis performed with any kind of magnification (loupes or microscope) presented a significantly better constant distance and symmetry between the stitches, whereas the operating microscope allowed a significantly lower number of backwall sutures $(\mathrm{P}<0.01)$ than the other methods. Comparisons of accuracy of the specimen showed significant superiority $(\mathrm{P}<0.01)$ of the operating microscope over the surgical loupes and simple naked-eye sutures (Table 2).

\section{DISCUSSION}

Microsurgical techniques have revolutionized many fields of modern surgery. In the history of microsurgery, the development and advancement of magnifying devices has always played a pivotal role. However, it is important and remarkable to note that the pioneers of microvascular surgery, such as Carrel [10], Hopfner [11], and Guthrie [12], did have the benefits of intraoperative magnification.

The history of optical magnification started with Lippershey and Janssen in 1590 when they first built a compound microscope by reversing a telescope [13]. Anton van Leeuwenhoek created the next hallmark in the development of magnifying devices, building powerful transportable microscopes with a basic design used without significant changes until the 20th century [14]. Carl Zeiss and Ernst Abbe combined their craftsmanship with their physics knowledge to create high quality microscopes with apochromatic lenses, which reduced chromatic aberrations even more than had been achieved with achromatic lenses; this was another important developmental step [15]. The microscope entered the operating theater when Nylen [16] treated a patient with chronic otitis media using a microscope. The event that marked the historical beginning of microvascular surgery was credited to Jacobson and Suarez [17] who first used a microscope for microvascular anastomosis. This hallmark innovation laid the foundation for all the great achievements in modern reconstructive microsurgery, such as thumb replantation [18] or free flap transplantation [19].

All of these remarkable developments made clear that adequate optical magnification is mandatory for successfully performing microsurgery. However, the application of such magnifying devices, such as the operating microscope or loupes, may pose an additional workload. Thus, surgery is performed without the aid of these devices in order to complete the operation within a reasonable amount of time. Typical examples of such clinical scenarios might include a cutting injury to the finger with laceration of the digital nerve that is sown in the emergency room or a severed digital artery during fasciectomy that has to be repaired. Although most readers know that such clinical problems call for surgery under magnification, at times external or internal causes prevent the surgeon from making the extra effort to use magnification. Another factor worth mentioning is that there is no legal requirement to use optical magnification under certain circumstances. Thus, it is not mandatory for a surgeon repairing a digital nerve to at least wear loupe magnification.

The results of our study showed that performing routine microsurgical tasks without any magnification device will produce significantly more mistakes. The current literature provides abundant information that learning and practicing microsurgery yields better results for a novice microsurgeon [20-24]. The goal and intention of our investigation was to show that different surgical tasks require different magnifying devices for achieving optimal results. To the best of our knowledge, this type of infor- 
mation has not been published yet. The results should serve as a guideline for new surgeons to understand what type of magnifying device is appropriate for a given surgical problem. During our basic microsurgical courses, we try to offer novice microsurgeons a sense and feeling for a delicate approach to specific tissues even if they do not pursue a career directly involved with microsurgery. We firmly believe that it is a great success when surgeons are aware of the fact that some clinical scenarios suggest a microsurgical approach for achieving superior results. This also means that a surgeon may refer patients to an appropriate institution for microsurgery.

We understand that the results of this study do not make the use of magnification devices mandatory or even legally binding for surgeons. However, this is the first experiment to demonstrate that the eye has limited abilities in certain surgical situations, and this limitation will lead to mistakes with a surgical outcome that is inferior to that which could be achieved with a magnification device. Therefore, our study should increase awareness of microsurgery and the intrinsic ties to adequate optical magnification in this surgical specialty.

By conducting practical experiments in a standardized manner with novice microsurgeons, we were able to prove that microsurgical success is directly related to optical magnification. The human eye's ability to discriminate potentially important anatomical structures is limited, which might be detrimental to clinical results. Although not legally mandatory, microsurgical operations such as reparative surgery after hand trauma should be conducted with magnifying devices for achieving an optimal patient outcome.

\section{REFERENCES}

1. Rohrich RJ, Rosen J, Longaker MT. So you want to be an innovator? Plast Reconstr Surg 2010;126:1107-9.

2. Kleinert HE, Kasdan ML. Restoration of blood flow in upper extremity injuries. J Trauma 1963;3:461-76.

3. Shenaq SM, Klebuc MJ, Vargo D. Free-tissue transfer with the aid of loupe magnification: experience with 251 procedures. Plast Reconstr Surg 1995;95:261-9.

4. Neaigus A. The network approach and interventions to prevent HIV among injection drug users. Public Health Rep 1998;113 Suppl 1:140-50.

5. Agarwal A. Visual acuity. In: Agarwal S, Agarwal A, Apple DJ, et al., editors. Textbook of opthalmology. New Delhi: Jaypee Brothers Medical Publishers Ltd.; 2002. p. 157-8.

6. Sucur D, Konstantinovic P, Potparic Z. Fresh chicken leg: an experimental model for the microsurgical beginner. $\mathrm{Br} \mathrm{J}$ Plast Surg 1981;34:488-9.
7. Schoffl H, Kropfl A. Alternatives to laboratory animals in microsurgical training. ALTEX 1994;11:32-9.

8. Schoffl H, Froschauer SM, Dunst KM, et al. Strategies for the reduction of live animal use in microsurgical training and education. Altern Lab Anim 2008;36:153-60.

9. Schoffl H, Hager D, Hinterdorfer C, et al. Pulsatile perfused porcine coronary arteries for microvascular training. Ann Plast Surg 2006;57:213-6.

10. Carrel D. Operative technic of vascular anastomoses and visceral transplantation. Lyon Med 1964;212:1561-8.

11. Hopfner E. Uber gefassnaht, gefasstransplantation und replantation von amputierten extremitaten. Arch Klin Chir 1903;70:417-71.

12. Guthrie CC. Blood-vessel surgery and its applications. New York: Edward Arnold-London; 1912.

13. Splinter R, Hooper BA. An introduction to biomedical optics. London: Taylor \& Francis; 2007.

14. van Zuylen J. The microscopes of Antoni van Leeuwenhoek. J Microsc 1981;121:309-28.

15. Louw DF, Sutherland GR, Schulder M. From microscopic to astronomic, the legacy of Carl Zeiss. Neurosurgery 2003; 52:668-74.

16. Nylen CO. The microscope in aural surgery, its first use and later development. Acta Otolaryngol Suppl 1954;116:226-40.

17. Jacobson JH, Suarez EL. Microsurgery in anastomosis of small vessels. Surg Forum 1960;11:243-5.

18. Komatsu S, Tamai S. Successful replantation of a completely cut-off thumb. Plast Reconstr Surg 1968;42:374-7.

19. Daniel RK, Taylor GI. Distant transfer of an island flap by microvascular anastomoses: a clinical technique. Plast Reconstr Surg 1973;52:111-7.

20. Chan WY, Matteucci P, Southern SJ. Validation of microsurgical models in microsurgery training and competence: a review. Microsurgery 2007;27:494-9.

21. Kazemi H, Rappel JK, Poston T, et al. Assessing suturing techniques using a virtual reality surgical simulator. Microsurgery 2010;30:479-86.

22. Carlson ML, Archibald DJ, Sorom AJ, et al. Under the microscope: assessing surgical aptitude of otolaryngology residency applicants. Laryngoscope 2010;120:1109-13.

23. Chan W, Niranjan N, Ramakrishnan V. Structured assessment of microsurgery skills in the clinical setting. J Plast Reconstr Aesthet Surg 2010;63:1329-34.

24. Chan WY, Figus A, Ekwobi C, et al. The 'round-the-clock' training model for assessment and warm up of microsurgical skills: a validation study. J Plast Reconstr Aesthet Surg 2010;63:1323-8. 